\title{
EVALUATION OF DIFFERENT CHANGE DETECTION TECHNIQUES IN FORESTRY FOR IMPROVEMENT OF SPATIAL OBJECTS EXTRACTION ALGORITHMS BY VERY HIGH RESOLUTION REMOTE SENSING DIGITAL IMAGERY
}

\author{
N. Amiri ${ }^{\text {a, }}$ \\ ${ }^{a}$ Dept. of Physical Geography and Ecosystem Science, Lund University, Lund, Sweden - \\ gif12nam@student.lu.se,n.amiri.1988@gmail.com
}

KEY WORDS: Change detection, pixel based, object based, VHRS optical images, Forestry

\begin{abstract}
:
Earth observations which are being useable by spatial analysis ability play an important role in detecting, management and solving environmental problems such as climate changes, deforestation, disasters, land use, water resource and carbon cycle. Remote sensing technology in combination with geospatial information system (GIS) can render reliable information on vegetation cover. Satellite Remote sensed data and GIS for land cover/use with its changes is a key to many diverse applications such as Forestry.

Change detection can be defined as the process of identifying differences in the state of an object or phenomenon by observing it at different times. The analysis of the spatial extent and temporal change of vegetation cover (Forest) by using remotely sensed data is critically importance to natural resource management sciences.

The main aim of this review paper is to go through the different change detection methods and algorithms based on very high resolution remote sensing imagery data, evaluate the quality of the spatial individual crown cover extraction in forests with high density, analyse, compare the results by optimized performance of control data for the same objects to provide the improvement in technique for detection and improve the mathematical sides of the change detection algorithms for high dense forests regions with different boundaries.
\end{abstract}

\section{INTRODUCTOIN}

Information on spatial distribution of forest cover classes is a primary and necessary requirement for sustainable forest management (Ghosh et al., 2014). The world`s forest covers are in the state of permanent flux at a variety of spatial and temporal scales (Coppin, 1996). The sustainability requirements of the present-day forest management are the accurate and continuously updated resource data of forest covers.

Forest inventory history starts with the history of the remote sensing, until vertical aerial photos become available in the 1930 `s, forest managers were totally depend upon resource information based on the ground (Coppin, 1996). Aerial Images have long provided the most common source of remotely sensed information for forest management (Kangas, 2006). Recently, remote sensing and satellite data have become a major application in forest inventory because of the repetitive coverage of the satellites at short intervals (Mas, 1999). While the concepts of change detection analysis is not new but evaluating the effects of new sensors and techniques for detect forest resources is still necessary (Forkuo, 2012).

With the marked improvement in spatial resolution of satellite imagery during the last decades, researchers have begun to explore utilization of satellite data. The increasing availability of data from high spatial resolution satellites for example, IKONOS, QuickBird, WorldView and GeoEye provides an ever broadening view compared to aerial photographs and low resolution images (Gougeon, 2003). Conventional low resolution images for crown cover detection methods are often an inefficient source of information due to the complexity of the forests, diversity of tree species and accessibility constraints to private property (Ward et al., 2007). However, the new paradigm of individual tree-based analysis is expected to lead to powerful remote sensing-based forest survey and management tools for individual tree crowns (Brandtberg et al., 2003). This paradigm commends a more object-oriented image analysis, one that shift from traditional pixel-based techniques and towards the detection of individual tree crowns (Gougeon \& Leckie, 2006). The object-oriented approach will reduce the local spectral variation caused by crown textures, gaps and shadows. In addition, any type of spatially distributed data such as elevation, intensity, vegetation index and population density can be used as input image segmentation to produce image objects (Ke et al., 2010; Suzuki, 2011).

Natural Resource Management, Planning and Monitoring programs in forests depends on accurate information about the land cover in a specific region. Satellite Imaging Corporation provides a large amount of remote sensing data at different spatial, spectral and temporal resolutions by using the appropriate combination of bands to bring out the geographical and manmade features for detecting changes. The analysis of very high resolution (VHR) images can help to update forest databases and to monitor the existence, rate and state of trees (Ardila et al., 2012).

"The type of the change detection method implemented can profoundly affect the qualitative and quantitative estimates of the disturbance"' (Singh, 1989). Even in the same environment, different approaches may yield different change detection maps. The selection of the appropriate method among various methods is also an important approach which takes on considerable significant. 
The main aim of this paper is to review the change detection techniques in two groups based on the unit of the image analysis. The first group is traditional/classical pixel based change detection techniques. The second group is the object based methods; creating crown covers as objects from images for further analysis of change detection. The paper is broadly based on three parts. The first part discussed about pixel based change detection methods in forestry then followed by object based detection algorithms and finally potentials of analysis by object based detection techniques from very high resolution satellite imagery. The paper is a review of commonly used object based techniques for detection of tree crown covers changes as spatial objects in forest vegetation, their applications and related issues.

\section{PIXEL BASED CHANGE DETECTION}

A pixel has been the basic and initial unit of image analysis since early apply of remote sensing data. During the change detection analysis spectral characteristics of each pixel has been measure and detect, mostly without considering the spatial context (Hussain et al., 2013). For Low resolution remote sensing satellite imagery pixel always has stand as a basic for analysis as it contains a large area on the ground.

Traditionally pixel based methods which they are mostly statistically oriented focus on spectral values not spatial (Hussain et al., 2013). These methods, based on the changes of a pixel without spatial context and statistical operators mostly used to evaluate the individual pixel. Different methods for pixel based approaches categorized in the figure 1 .

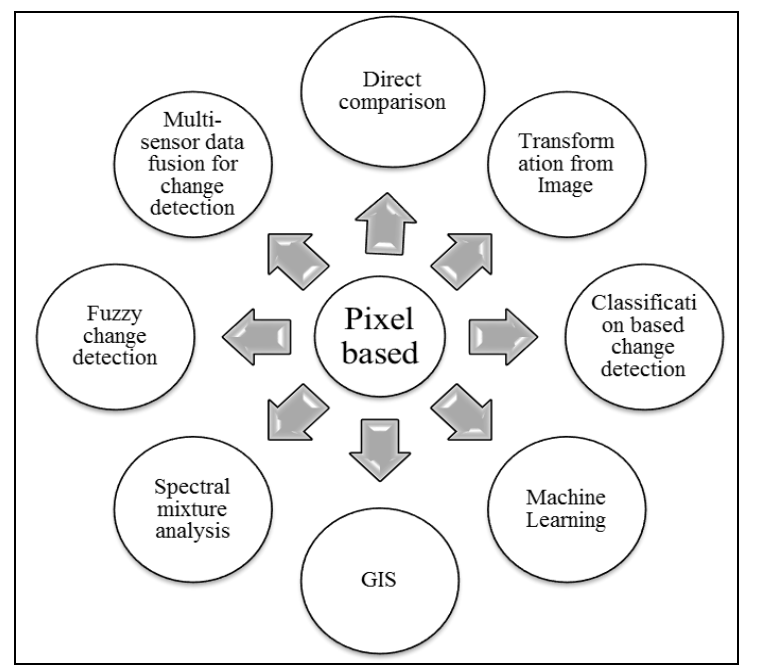

Figure 1, Overview of different pixel based change detection methods

Threshold value to differentiate in forests is used in most detection algorithms and selecting a suitable threshold for identify is not easy (Lu et al., 2004), especially in the case of unsupervised techniques with lack of ground truth points. Test a continues threshold instead of discrete as a calibration method can reduce the limitations of traditional methods. Some approaches to improve the threshold selection are fuzzy set and fuzzy membership functions (Metternicht, 1999); Bayes rule analysis (Bruzzone et al., 2000) and the use of existing objects for automated threshold calculation (Bouziani et al., 2010). However, spatial aspects and relations of objects in the real world could not be modelled in these analysis of pixel based and leads to noisy results (Ke et al., 2010).

\section{ISSUES WITH TRADITIONAL TECHNIQUES}

Currently, researches focus on accurate derivation of forest inventory parameters from the delineated tree crowns, such as tree volume estimates, specious composition and individual tree health information to improve automated forest inventory and analysis. The traditional pixel base detection methods are classifying the each pixel in the image without regard to neighbour pixels. Pixel is not the optimal spatial unit for mapping the landscape especially in high resolution satellite images because of mixed pixel problem as one of the major error sources in detection (Blaschke, 2010).

Also, one of the main problems of pixel based approaches was related to threshold value for change and unchanged areas. However, most of the methods based on a critical selection value, threshold. This value is the relationship between image resolution, forest condition and tree crown size and effected the result of detection accuracy (Ke et al., 2011). Threshold value to differentiate crown covers from each other in forests is used in most detection algorithms. Selecting a suitable threshold to identify the individual crown cover boundaries is not easy $(\mathrm{Lu}$ et al., 2004).

Geometric co-registration errors and results of object extraction (such as classification and pattern recognition) are the two main factors which greatly affect the results of change detection in traditional methods (Xiaodong et al., 2006). For the purpose of more correct pixel-pixel analysis, a geometric co-registration operation is necessary to make geometric information of the images to stay the same. The precision of geometric correction has an important influence on the change detection results, since a low precision could lead to a mass of false changes (Rogan et al., 2004). Initially Townshend et al. (1992) quantificationally researched the influence of the geometric correction error on change detection results using Landsat MSS satellite data.

\section{OBJECT BASED CHANGE DETECTION}

As an alternative to traditional approaches, object-based classification was introduced and has been widely used to solve the problems associated with the high spatial resolution domain which is not easy by pixel based methods (Ke et al., 2010). In the object based classification, the image-objects are extracted and then classification by supervised or unsupervised algorithms (Hussain et al., 2013). The object based image analysis has been used in the direction of reducing the effects of higher spectral variability; reduce the effects of geo-referencing and acquisition characteristics (Hussain et al., 2013). This method allows the segmentation and extraction of features from VHR data and also facilitates the integration of raster-based processing and vector-based GIS (Blaschke, 2010). The unit of the measurement consists of texture, shape, spatial resolution with neighbour objects and ancillary spatial data. The significant approach of the method for improves the classification, image segmentation and object hierarchy development. Segmentation process partitions an image into homogenous objects which are spectrally similar and spatially adjacent (Chen et al., 2012). 
More recently, object-based methods have been proposed for tree cover detection to combine the contextual analysis of visual interpretation with the quantitative aspect of pixel-based approaches (Desclee et al., 2006). The interest for object-based methods in forestry has increased with the improvements in image segmentation techniques (Mäkelä, 2001). Image segmentation is the division of the satellite image into spatially continuous and homogeneous regions, which named as objects. The main advantage of object-based methods is the incorporation of contextual information in the forest inventory and detection analysis (Flanders, 2003).

The history of research into automatic individual tree detection from digital imagery dates back to the mid-1980s. One of the earliest examples was the research which was presented by Pinz (Pinz, 1991) using the Vision Expert to locate the center of a crown and estimate the crown radius by searching for local brightness maxima in smoothed aerial images with a $10 \mathrm{~cm}$ pixel size. In the mid-1990s, (Gougeon, 1995a) presented a valley-following and rule-based algorithm to delineate fully coniferous tree crowns by following valleys of shadows between tree crowns using $36 \mathrm{~cm}$ ground sampled distance (GSD) digital aerial imagery (Ke et al., 2011). In the same period of time, multiple scale analysis was applied on higher resolution imagery to estimate tree-crown area (Brandtberg, 1998), and model-based template matching techniques were introduced to recognize individual trees (Pollock, 1996).

Later, methods such as Multidimentional Temporal Feature Space Analysis, Composite Analysis, Image Differencing, Image Rationing, Change Vector Analysis, Image Regression, multi temporal Biomass Index were introduced for change detection purposes. However, the methods reported are different in terms of the type of algorithms which is used, the conditions of specific study area, the types of applicable imagery and the accuracy of the evaluation methods considered (Culvenor, 2002). Additionally, the increased speed found in modern computer systems has facilitated, the development of digital image analysis for automated recognition of specific object characteristics (Ke et al., 2011). Different object based change detection methods presented in the figure 2 .

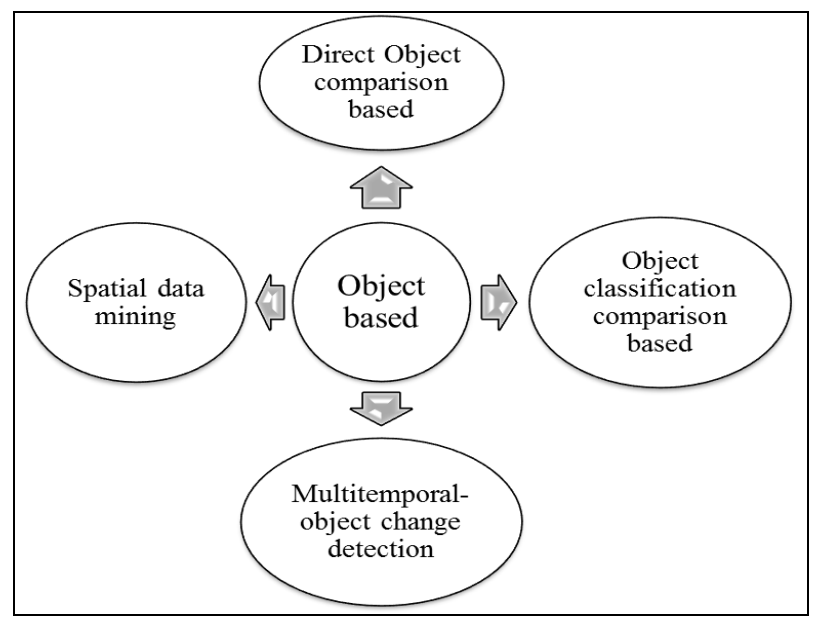

Figure 2, Overview of different object based change detection techniques

The VHR satellite images provide spectral signature of the individual tree canopies as objects, an important limitation in the forest inventory studies still remains which is lack of high geometric detail (peaks and valleys) to explain the height, structure and size of crowns (Hu et al., 2012). However, similarity of spectral signatures for different tree species, as well as assemblage of tree crowns with little to no inter crown distance and occurrence of over crown canopy, increase the challenges for successful tree cover change mapping from optical very high resolution remote sensing data (Ghosh et al., 2014).

\section{CHALLENGES FOR OBJECT BASED APPROACHES}

Most object based algorithms for crown cover were applies on vertical aerial imagery, there are a few studies utilized very high resolution satellite imagery (Ke et al., 2011).The best classification principle of "homogeneity within objects, heterogeneity among objects" is the important factor in the decision process of the best scale for segmentation(Lian et al., 2011). Because of more details in the higher spatial resolution data, the greater the scale will be and the better effect of image segmentation and vice versa but not necessarily the better classification accuracy.

Moreover, the segmentation algorithms affect the geometry of the result objects which need appropriate solution to keep the relation of the extracted objects from two different times for detecting changes (Hussain et al., 2013). Also, there are not strongly reliable methods for accuracy assessment of object based detection methods yet. Most of the accuracy evaluation based on pixel based methods (Ghosh et al., 2014).

\section{FUTURE OF CHANGE DETECTION BY REMOTE SENSING}

Take a look to the automatically selecting the appropriate parameters for object extraction, defining standard frame work for object comparison and the accuracy assessment of change detection results of object based methods are necessary for future improvements. Other techniques employing advanced computational and data processing algorithms such as AI, knowledge-based expert system, agent-based models and machine learning algorithms represent new directions in change detection studies. Optimal segmentation scale as a range is also one of the parameters which affected the results of object based change detection methods.

The development of digital change detection techniques had limitations due to small capacities of data processing technology and followed closely the development of computer technologies which improves data processing. In the future, these fields will continue evolving parallel and new developments are expected with the development of computer technologies and artificial intelligence. However, in practice, the selection of the most suitable method or algorithm is not easy in many cases (Lu et al., 2004).

During the last two decades, a large variety of tree crown cover detection algorithms has been developed and processed. The advantages/disadvantages of a particular method can greatly affect the result of tree crown cover detection, therefore for a specific application could affect derivation of descriptive parameters. Even though, in the same environment, different purposes may yield to different results. Therefore, the selection of an appropriate algorithm has considerable significance based on the approach (Ke et al., 2011). 
With the development of remote sensing technology and data and potentials of using multidate radar imagery, which recently captured the attention of researchers (Coppin, 1996) the object based methods for change detection has witnessed their ability and reliability. This approach is sustainable evaluation for traditional pixel based methods in compare with object based change detection methods.

\section{SUMMARY}

The vegetation change detection process divided in to preprocessing, selecting the appropriate technique and accuracy assessment (Çilsever et al., 2012 ). Remote sensing techniques provide a potentially low-cost alternative to field-based assessment, but require the development of methods to easily and accurately extract the required information (King, 2002). Automatic tree crown cover detection and delineation algorithms may be an effective means to accomplish this task. Since the reviewed algorithms were developed for specific site conditions, used different types of imagery and evaluated with different accuracy assessment approaches, it is difficult to compare their performance (Ke et al., 2011). However, improving the current methods and algorithms for crown cover delineation and detection by ancillary data will develop to become suitable to be used in different forest conditions and image types.

\section{Acknowledgement}

This review research is done by support of Prof. Dr. Lars Eklundh from Department of Physical Geography and Ecosystem Science, Lund University, Sweden and Dr. Y.A. (Yousif) Hussin from Faculty of Geo-Information science and Earth observations, ITC, University of Twente, the Netherlands.

\section{References}

Ardila, J. P., Bijker, W., Tolpekin, V. A., \& Stein, A. (2012). Quantification of crown changes and change uncertainty of trees in an urban environment. ISPRS Journal of Photogrammetry and Remote Sensing, 74(0), 41-55. doi: http://dx.doi.org/10.1016/j.isprsjprs.2012.08.007

Blaschke, T. (2010). Object based image analysis for remote sensing. ISPRS Journal of Photogrammetry and Remote Sensing, $\quad 65(1), \quad 2-16 . \quad$ doi: http://dx.doi.org/10.1016/j.isprsjprs.2009.06.004

Bouziani, M., Goita, K., \& He, D. C. (2010). Automatic change detection of buildings in urban environment from very high spatial resolution images using existing geodatabase and prior knowledge. Isprs Journal of Photogrammetry and Remote Sensing, 65(1), 143-153. doi: DOI 10.1016/j.isprsjprs.2009.10.002

Brandtberg. (1998). Automated delineation of individual tree crowns in high spatial resolution aerial images by multiplescale analysis. Machine vision and Applications, 11.

Brandtberg, \& T. (2003). Detection and analysis of individual leaf-off tree crowns in small footprint, high sampling density lidar data from the eastern deciduous forest in North America. Remote Sensing of Environment, 85(3), 290-303. doi: 10.1016/s0034-4257(03)00008-7

Bruzzone, L., \& Prieto, D. F. (2000). Unsupervised change detection in multisource and multisensor remote sensing images. Igarss 2000: Ieee 2000 International Geoscience and Remote Sensing Symposium, Vol I - Vi, Proceedings, 24412443.

Chen, G., Hay, G. J., Carvalho, L. M. T., \& Wulder, M. A. (2012). Object-based change detection. International Journal of Remote Sensing, 33(14), 4434-4457. doi: 10.1080/01431161.2011.648285

Çilsever, M., \& Ünsalan, C. (2012 ). Two-Dimensional Change Detection Methods. 76.

Coppin, P. R., \& Bauer, M. E., . (1996). Change Detection in Forest Ecosystems with Remote Sensing Digital Imagery. Remote Sensing Reviews, 13.

Culvenor, D. S. (2002). TIDA: an algorithm for the delineation of tree crowns in high spatial resolution remotely sensed imagery. Computers \& Geosciences, 28(1), 33-44. doi: http://dx.doi.org/10.1016/S0098-3004(00)00110-2

Desclee, B., Bogaert, P., \& Defourny, P. (2006). Forest change detection by statistical object-based method. Remote Sensing of Environment, 102(1-2), 1-11. doi: 10.1016/j.rse.2006.01.013

Flanders, D., Hall-Beyer, M., Pereverzoff, J. . (2003). Preliminary evaluation of eCognition object-based software for cut block delineation and feature extraction. Canadian Journal of Remote Sensing, 29(4).

Forkuo, E., K., Frimpong, A. (2012). Analysis of Forest Cover Change Detection International Journal of Remote Sensing Applications 2(4).

Ghosh, A., Fassnacht, F. E., Joshi, P. K., \& Koch, B. (2014). A framework for mapping tree species combining hyperspectral and LiDAR data: Role of selected classifiers and sensor across three spatial scales. International Journal of Applied Earth Observation and Geoinformation, 26(0), 49-63. doi: http://dx.doi.org/10.1016/j.jag.2013.05.017

Gougeon. (1995a). A Crown-Following Approach to the Automatic Delineation of Individual Tree Crowns in High Spatial Resolution Aerial Images. Canadian Journal of Remote Sensing, 21 .

Gougeon. (2003). The Individual Tree Crown (ITC) Approach to Forest Inventories: Satellite and Aerial Sensor Considerations.

Gougeon \& Leckie. (2006). The Individual Tree Crown Approach Applied to Ikonos Images of a Coniferous Plantation Area Photogrammetric Engineering \& Remote Sensing, 72(11), 1287-1297.

Hu et al. (2012). Individual Urban Tree Species Classification Using Very High Spatial Resolution Airborne Multi-Spectral Imagery Using Longitudinal Profiles. Remote Sensing, 4(12), 1741-1757. doi: 10.3390/rs4061741 
Hussain, M., Chen, D., Cheng, A., Wei, H., \& Stanley, D. (2013). Change detection from remotely sensed images: From pixel-based to object-based approaches. ISPRS Journal of Photogrammetry and Remote Sensing, 80(0), 91-106. doi: http://dx.doi.org/10.1016/j.isprsjprs.2013.03.006

Kangas, A. M. M. (2006). Forest inventory methodology and applications, from http://site.ebrary.com/id/10182665

Ke, Y., \& Quackenbush, L. J. (2011). A review of methods for automatic individual tree-crown detection and delineation from passive remote sensing. International Journal of Remote Sensing, 32(17), 4725-4747. doi: $10.1080 / 01431161.2010 .494184$

Ke, Y., Quackenbush, L. J., \& Im, J. (2010). Synergistic use of QuickBird multispectral imagery and LIDAR data for objectbased forest species classification. Remote Sensing of Environment, 114(6), 1141-1154. doi: 10.1016/j.rse.2010.01.002

King, D. J., Bell, F.W. , Pitt, D.G. . (2002). Automated tree crown detection and delineation in high-resolution digital camera imagery of coniferous forest regeneration. Remote Sensing of Environment 82 322-334.

Lian, L., \& Chen, J. (2011). Research on Segmentation Scale of Multi-Resources Remote Sensing Data Based on ObjectOriented. Procedia Earth and Planetary Science, 2(0), 352357. doi: http://dx.doi.org/10.1016/j.proeps.2011.09.055

Lu, D., Mausel, P., Brondizio, E., \& Moran, E. (2004). Change detection techniques. International Journal of Remote Sensing, 25(12), 2365-2407. doi: Doi 10.1080/0143116031000139863

Mäkelä, H., Pekkarinen, A. . (2001). Estimation of timber volume at the sample plot level by means of image segmentation and Landsat TM imagery. [Journal ]. Remote Sensing of Environment, 77.

Mas, J. F. (1999). Monitoring land-cover changes: a comparison of change detection techniques. International Journal of Remote Sensing, 20(1), 139-152. doi: Doi $10.1080 / 014311699213659$

Metternicht, G. (1999). Change detection assessment using fuzzy sets and remotely sensed data: an application of topographic map revision. Isprs Journal of Photogrammetry and Remote Sensing, 54(4), 221-233. doi: Doi 10.1016/S09242716(99)00023-4

Pinz, A. (1991). A computer vision system for recognition of trees in aerial photographs. In Proceedings of the International Assosiation of pattern recognition Workshop, 111-124.

Pollock, R. J. (1996). The automatic recognition of individual trees in aerial images of forests based on a synthetic tree crown image model. [PHD dissertation].

Rogan, J., \& Chen, D. (2004). Remote sensing technology for mapping and monitoring land-cover and land-use change. Progress in Planning, 61(4), 301-325. doi: 10.1016/s03059006(03)00066-7

Singh, A. (1989). Review Article Digital change detection techniques using remotely-sensed data. International Journal

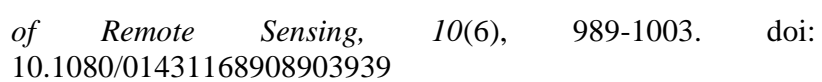

Suzuki, R., et al. (2011). NDVI responses to the forest canopy and floor from spring to summer observed by airborne spectrometer in eastern Siberia. Remote Sensing of Environment, 115(12), 3615-3624. doi: http://dx.doi.org/10.1016/j.rse.2011.08.022

Ward, K. T., \& Johnson, G. R. (2007). Geospatial methods provide timely and comprehensive urban forest information. Urban Forestry \& Urban Greening, 6(1), 15-22. doi: 10.1016/j.ufug.2006.11.002

Xiaodong, Z., Gong, J., Y.,, \& Deren, L. (2006). A strategy of change detection based on remotely sensed imagery and GIS data. . [Commission VII, WG VII/5]. [973 Program of China]. Guangdong Natural Science Foundation, 6. 